\title{
Multiple sclerosis presenting with progressive visual
}

\section{failure}

\author{
IAN EC ORMEROD, WI McDONALD \\ From the University Department of Clinical Neurology, The National Hospital for Nervous Diseases, Queen \\ Square, London UK
}

SUMMARY Progressive visual failure as the presenting feature of multiple sclerosis is described in five patients. The clinical features did not permit a distinction from visual loss due to compression. The finding of oligoclonal bands in the CSF at presentation is a useful pointer to the diagnosis, but is not specific and full investigation to exclude treatable causes of visual loss is essential.

Acute remitting unilateral optic neuritis is a common mode of presentation of multiple sclerosis. By contrast, progressive visual failure rarely heralds the onset of the disease although it may develop during the course of established multiple sclerosis. ${ }^{1-4}$ Schlossman and Philips ${ }^{1}$ observed it in seven of 57 patients with multiple sclerosis who had visual symptoms, and Kahana ${ }^{2}$ found it in 25 of 185 patients with multiple sclerosis and clinical signs of optic nerve involvement. Ashworth ${ }^{3}$ described nine patients with probable or possible multiple sclerosis who developed "chronic retrobulbar neuritis" or "chronic chiasmal neuritis". In none of these reports is reference made to progressive visual failure as the presenting feature of multiple sclerosis. In this paper we report five such cases with a view to identifying features which might allow an early distinction from compression of the anterior visual pathways.

\section{Patients}

The patients were originally referred for investigation of progressive visual loss. In making the diagnosis of multiple sclerosis we have used the recently published criteria which include the results of laboratory investigations. ${ }^{5}$

Case 1: A 44-year-old man presented in 1973 with bilateral progressive visual failure of 6 months duration. The right eye only had been affected at first but after 4 months the left eye also became involved. There was slight discom-

Address for reprint requests: Prof WI McDonald, the National Hospital for Nervous Diseases, Queen Sq, London WC1N 3BG, UK.

Received 24 January 1984. Accepted 23 February 1984 fort in the right eye, occurring for a few days shortly after the onset of the symptoms.

On examination the visual acuities on the right and the left (VAR, VAL) were reduced to counting fingers at 50 $\mathrm{cm}$. In both eyes there was a central scotoma and an afferent pupillary defect. The right fundus showed optic atrophy. The remainder of the examination was normal. A lumbar puncture revealed normal CSF; protein electrophoresis was not then available.

In 1980,7 years after the initial presentation, he suffered an episode of diplopia and dysarthria of sudden onset which recovered over 2 weeks. In 1981 he was readmitted following further deterioration of the vision in the left eye. On examination the acuities were VAR 3/60 and VAL, perception of light. There was a central scotoma on the right and bilateral optic atrophy: Motor examination showed ataxia and pyramidal signs in all limbs. A CT scan showed mild cerebral atrophy with small areas of low attenuation in both hemispheres. The CSF protein was elevated at $0.96 \mathrm{~g} / \mathrm{l}$ with no oligoclonal bands found on polyacrylamide gel electrophoresis.

A diagnosis of clinically definite multiple sclerosis was made on the basis of the remitting episode of brainstem disturbance in a setting of visual loss with additional clinical and CT evidence of multiple lesions. In 1983 the patient died at another hospital. No post mortem examination was performed.

Case 2: A 33-year-old female presented in 1976 with a 3 month history of visual impairment. She had initially noticed difficulty in reading fine print and then progressive failure of vision in the right eye. Two months later the sight in the left eye also became blurred. Investigations, including bilateral carotid arteriograms and an air encephalogram were performed but no cause was found. The visual acuity fell further and there was intermittent retro-ocular pain on both sides. There had been some menstrual irregularity.

On examination 7 months after the first symptom, the 
visual acuities were VAR, no perception of light and VAL counting fingers. There was bilateral severe optic atrophy. Motor examination showed a mild left pyramidal deficit with brisk reflexes and an equivocal plantar response.

The auditory evoked potentials (AEPs) and somatosensory evoked potentials (SEPs) were normal. Repeat carotid arteriograms and air encephalography were normal as was a CT scan. Lumbar puncture showed a CSF with no cells, normal protein, but an oligoclonal pattern on electrophoresis.

In 1977 the visual acuities remained unchanged. She had developed nystagmus on left lateral gaze and upbeat nystagmus on upgaze. The left sided pyramidal signs were unchanged. Early in 1983 the acuities were VAR no perception of light, VAL perception of light. There was a spastic paraparesis with bilateral extensor plantar responses. The leg weakness progressed over the following months and in June 1983 she began to experience urgency of micturition. She was readmitted to hospital in November 1983 and with physiotherapy there was some improvement in the power of the legs and also improvement in bladder function.

A diagnosis of clinically definite multiple sclerosis was made on the grounds of a partially remitting spinal cord disorder in association with clinical evidence of multiple lesions and oligoclonal bands in the CSF.

Case 3: This 51-year-old male presented in 1976 with a 3 month history of progressive simultaneous deterioration of vision in both eyes. On examination the visual acuities were VAR 6/12 and VAL counting fingers at 1 metre. Over the following 2 months VAR fell to $6 / 60$ and examination showed bilateral central scotomata and bilateral optic atrophy. Investigations, including air encephalography and lumbar puncture were normal, although the CSF was not examined for oligoclonal bands.

His condition remained unchanged for 3 years. He then began to notice persistent tingling in his extremities and difficulty in identifying objects with his right hand. There was no remission in these symptoms, although it was noted that the tingling became worse with exertion. On examination in 1982, the acuities were VAR 6/60, VAL 6/60 with bilateral central scotomata and optic atrophy. There was ataxia in the lower limbs with an extensor plantar response on the right. Sensory examination showed absent vibration sense below the costal margin and astereognosis in the right hand.

SEPs to median nerve stimulation showed normal plexus responses (N9), absent cervical responses bilaterally (N11, $\mathrm{N} 13$ and N14) and delayed cortical responses (N20). These findings were reported as consistent with a cervical cord lesion (Dr NMF Murray). A CT scan was normal. Lumbar puncture showed a CSF protein of $0.5 \mathrm{~g} / 1$ with an oligoclonal pattern on electrophoresis.

A diagnosis of laboratory supported definite multiple sclerosis was made on the grounds of the progressive history, signs and laboratory evidence of multiple lesions and oligoclonal bands detected on polyacrylamide gel electrophoresis of CSF protein.

Case 4: A 40-year-old male presented in 1981 with a 4 month history of visual impairment. He initially suffered episodes of bilaterally blurred vision of sudden onset, coming on over a few seconds and lasting 30-60 minutes. Over a period of 3 to 4 weeks about 10 of these attacks had occurred at decreasing intervals. At this time he noticed that his vision did not return to normal between episodes. The course then was of progressive visual impairment affecting the left eye mainly. He also noticed a deterioration in his appreciation of colours.

On examination the visual acuities were VAR 6/9, VAL $6 / 60$. Colour vision was reduced with $2 / 17$ Ishihara plates correctly interpreted on the right and none on the left. The visual fields showed a superior temporal altitudinal defect on the right and a dense left central scotoma. There was a left relative afferent pupillary defect with optic atrophy and slits in the nerve fibre layer on the left on fundoscopy. The remainder of the examination was normal. Visual evoked potentials (VEPs) were bilaterally abnormal being almost absent from the left and markedly degraded from the right. There were no central field P100 responses. Bilateral carotid arteriograms, air encephalography and CT scan were normal. The CSF contained no cells but a protein of $0.82 \mathrm{~g} / \mathrm{l}$ and oligoclonal banding on electrophoresis.

Late in 1982 he began to suffer some unsteadiness of his gait but had no clearly defined episode of ataxia. There was also a mild, but progressive, weakness of both legs. Over the following 12 months further symptoms developed; he complained of persistent urgency and hesitancy of micturition and difficulty in maintaining an erection. He had an episode of numbness affecting the left side of the face, with objective signs of sensory impairment, lasting approximately 4 weeks.

On examination in 1983 the visual acuities were VAR $6 / 12$, VAL $6 / 12$, with central scotomata and optic atrophy affecting both eyes. The eye movements were dysmetric. The legs were ataxic with brisk reflexes and extensor plantar responses.

A diagnosis of clinically definite multiple sclerosis was made on the grounds of the remitting episodes of neurological disturbance, clinical evidence of multiple lesions and oligoclonal bands on analysis of the CSF.

Case 5: This man was first seen in the outpatients department in October 1981, when he was aged 23 years. Early in 1981 he suffered right-sided headaches and right retrobulbar pain, which lasted about a week and was not associated with an impairment of vision. In May of that year he noted his vision was blurred and also found that brightly illuminated objects would transiently shimmer if he stared intently at them. He consulted his optician who prescribed glasses and his vision was apparently corrected to normal. In August of 1981 when he happened to close his left eye he found that the sight in the right eye was markedly impaired particularly in the temporal field. Over the next 2 months, the vision deteriorated further in the right eye and central vision in the left eye also became progressively impaired.

On examination in the outpatients department, the acuities were VAR $<6 / 60$ and VAR 6/9. He was unable to read any of the Ishihara colour plates with either eye. The right eye had a centro-caecal scotoma and optic atrophy.

Radiological examination of the optic foramina, tomog- 
raphy of the optic canals and CT scan of brain and orbits were all normal. The VEPs showed bilateral absent macular (P100) responses but preserved paramacular responses characteristic of central scotomata. ${ }^{6}$ The CSF protein was $0.3 \mathrm{~g} / 1$ with an oligoclonal pattern on electrophoresis.

In June 1982 he suffered an episode of numbness and tingling affecting his right leg which subsequently resolved. His vision had remained unchanged but then deteriorated further in August 1982. Examination at that time showed the acuities to be VAR finger counting at 1 metre, VAL $6 / 24$. Both eyes showed central scotomata and optic atrophy.

In June 1983 he developed an internuclear ophthalmoplegia which subsequently resolved. The vision had continued to deteriorate being reduced to VAR perception of light, VAL 6/36.

A diagnosis of clinically definite multiple sclerosis was made on the basis of an episode of remitting sensory disturbance, an internuclear ophthalmoplegia, clinical and paraclinical evidence of further lesions and an oligoclonal pattern on CSF protein electrophoresis.

\section{Discussion}

These five cases of progressive visual failure which proved to be due to multiple sclerosis constitute an uncommon problem even in units to which considerable numbers of patients with visual loss are referred. Diagnosis presented considerable difficulty and was delayed for 3 years in Case 3 and 7 years in Case 1. Most familial, toxic, infective and inflammatory causes were readily excluded and the most important differentiation was from tumour. Comparison of the clinical features of these five cases with those of others presenting in a similar way has not allowed us to identify any characteristic clinical features which permit a reliable distinction between multiple sclerosis and compression. Pain is not invariable in demyelinating optic neuritis and is occasionally encountered when the optic nerve is compressed by tumour, aneurysm or mucocele ${ }^{7}$ of the sphenoid sinus. The field defect is unhelpful: central scotoma occasionally occurs with compression.

Our experience suggests that investigation should proceed along two lines from the start. First, a mass must be actively sought by high resolution CT scanning with enhancement, both of the chiasmal region and the orbits, with reconstruction of the optic nerves throughout their length, including the portions in the optic canals. Plain radiographs should also be taken since they may reveal more clearly than the CT scans an increase in bony density in association with meningioma en plaque involving the sphenoid wings. X-ray tomography of the optic canals may also be required since it may reveal expansion more easily than computed tomography, although the capacity of the latest generation of scanners in this respect has still to be evaluated. The scanner may give a clue to sinus disease accompanying a mucocele ${ }^{8}$ or to a vascular abnormality such as aneurysm or ectasia of the cerebral arteries causing visual loss. ${ }^{9}$ It cannot however exclude vascular lesions and when in doubt arteriography is necessary.

The second line of investigation should be directed towards finding other evidence of multiple sclerosis. The VEP is of little use in the present context. Although a delay in a well formed $\overline{\mathrm{P} 100}$ is characteristic of multiple sclerosis it is not diagnostic of it: we have seen it with compression of the intracranial optic nerve by aneurysm and meningioma.? Experimental work suggests that in these circumstances compression induces diffuse demyelination in the optic nerves. ${ }^{70}$ Conversely, the characteristic VEP features in compression of the visual pathways are changes in waveform and distribution, reflecting the location of the mass. We have however seen such changes in multiple sclerosis." Abnormalities in the AEP and SEP provide evidence of lesions in the corresponding pathways, but cannot of themselves provide clinching evidence for the diagnosis of multiple sclerosis in the present context. The finding of oligoclonal bands in the CSF provides good evidence of an immunological abnormality in relation to the nervous system which is most often due to multiple sclerosis and was present while the symptoms were confined to the visual system in two of our patients. Again, oligoclonal bands are not found solely in multiple sclerosis. ${ }^{12}$

We conclude that progressive visual failure should only be attributed to multiple sclerosis after other causes have been rigorously excluded and the criteria for clinically definite multiple sclerosis strictly fulfilled.

We are grateful to Dr CJ Earl and Professor RW Gilliatt for permission to report cases under their care.

\section{References}

' Schlossman A, Philips CC. Optic neuritis in relation to demyelinating disease. Am J Ophthalmol 1954; 37:487-94.

${ }^{2}$ Kahana E, Leibowitz U, Fishback N, Alter M. Slowly progressive and acute visual impairment in multiple sclerosis. (Minneap) Neurology 1973;23:729-33.

${ }^{3}$ Ashworth B. Chronic retrobulbar and chiasmal neuritis. Br J Ophthalmol 1967;51:698-702.

${ }^{4}$ Scott GI. Ophthalmic aspects of demyelinating diseases. Proc Roy Soc Med 1961;54:38-42.

${ }^{5}$ Poser CM, Paty DW, Scheinberg L, et al. New diagnostic criteria or multiple sclerosis: guidelines for research protocols. Ann Neurol 1983;13:227-31. 
- Halliday AM, Barrett G, Blumhardt LD, Kriss A. The macular and paramacular subcomponents of the pattern evoked response. In: Lehmann D, Callaway E, eds. Human Evoked Potentials. Plenum Publishing Corporation, 1979:135-51.

${ }^{7}$ McDonald WI. The symptomatology of tumours of the anterior visual pathways. The 1981 Silversides lecture. Can J Neurol Sci 1982;9:381-90.

${ }^{8}$ Lew D, Southwick FS, Montgomery WW, et al. Sphenoid sinusitis. A review of 30 cases. New Engl J Med 1983;309:1149-54.

9 Yu YL, Moseley IF, Pullicino P, McDonald WI. The clinical picture of ectasia of the intracerebral arteries. J Neurol Neurosurg Psychiatry 1982;45:29-36.

${ }^{10}$ Clifford Jones RE, Landon DN, McDonald WI. Demyelination during optic nerve compression. $J$ Neurol Sci 1980;46:239-43.

"McDonald WI. The role of evoked potentials in the diagnosis of multiple sclerosis. In: Bauer HJ, Poser S, Ritter G, eds. Progress in Multiple Sclerosis Research. Berlin: Springer Verlag, 1980:564-8.

12 Thompson EJ, Kaufman P, Shortman RC, Rudge P, McDonald WI. Oligoclonal immunoglobulins and plasma cells in spinal fiuid of patients with multiple sclerosis. Br Med J 1979; 1:16-17. 